\title{
Spontaneous Eye Blink Rate during the Working Memory Delay Period Predicts Task Accuracy
}

\author{
Jefferson Ortega ${ }^{1}$, Chelsea Reichert Plaska ${ }^{1,2}$, Bernard A. Gomes ${ }^{3}$, \\ Timothy M. Ellmore ${ }^{1,2^{*}}$ \\ ${ }^{1}$ Department of Psychology, The City College of the City University of New York, New York, USA \\ ${ }^{2}$ Behavioral and Cognitive Neuroscience Program, The Graduate Center of the City University of New \\ York, New York, USA \\ ${ }^{3}$ Department of Neurosurgery, Cedars-Sinai Medical Center, Los Angeles, California, USA
}

${ }^{*}$ Corresponding Author:

Timothy M. Ellmore, Ph.D.

Professor

The City College of New York

Department of Psychology, NAC 7/120

160 Convent Avenue

New York, NY 10031

e-mail: tellmore@ccny.cuny.edu

phone: 212-650-5714

fax: 212-650-5659 


\begin{abstract}
Changes in the spontaneous eye blink rate (sEBR) have been linked to a range of cognitive functions including attention and learning and memory, specifically working memory (WM). A growing body of literature also links sEBR to striatal dopamine (DA) activity with schizophrenia and Parkinson's disease showing increases and decreases respectively in sEBR relative to healthy subjects. Dopamine plays a role in working memory since dopamine neurons project to prefrontal cortex (PFC) through the mesocortical dopamine pathway and their activity has been implicated in WM maintenance, gating, and updating. A weakness of past studies investigating sEBR and WM is that correlations have been reported using blink rates taken at baseline either before or after performance of the tasks used to assess WM. The goal of the present study was to understand how fluctuations in sEBR during the different phases of a Sternberg visual WM task predict task accuracy. In two experiments, where recordings of sEBR were collected inside and outside of a magnetic resonance imaging bore, we observed sEBR to be positively correlated with WM task accuracy during the WM delay period. We also found significant task-related modulation of sEBR, including higher sEBR during the delay period compared to rest, and lower sEBR during task phases (e.g., stimulus encoding) that place high demands on visual attention. Taken together, these results provide further evidence that SEBR could be an important predictor of WM task performance with the changes during the delay period suggesting a role in WM maintenance. Additional analyses that explored the non-linear relationship between sEBR and WM task accuracy found that a 2nd order relationship explained some of the variance, but the fit was not significant. The relationship of sEBR to DA activity and the influence of DA during WM maintenance is discussed.
\end{abstract}

Keywords: Spontaneous eye blink rate, working memory, delay period, dopamine, attention 


\section{Introduction}

The healthy human blinks around 15-20 times per minute (Tsubota et al., 1996), however the precorneal tear film, which lubricates the eye, only begins drying up approximately 25s after a blink ends (Norn, 1969). This suggests that we blink more often than needed to maintain a lubricated precorneal tear film. Previous research has found task-related modulation of spontaneous eye blink rate (sEBR), which indicates that blinking could be reflective of cognitive factors (Oh, Jeong, \& Jeong, 2012; Siegle, Ichikawa, \& Steinhauer, 2008). For example, reading is accompanied by low levels of sEBR, while high levels of sEBR have been reported during conversation (Bentivoglio et al., 1997). More recent studies have found that sEBR correlates with attentional load and fatigue (Maffei \& Angrilli, 2018), attentional control (Colzato, van den Wildenberg, van Wouwe, Pannebakker, \& Hommel, 2009; Unsworth, Miller, \& Robison, 2019), can track working memory updating and gating (Rac-Lubashevsky, Slagter, \& Kessler, 2017), and can predict differences in exploration during reinforcement learning (Van Slooten, Jahfari, \& Theeuwes, 2019). In addition, a growing body of literature continues to provide evidence supporting sEBR as an effective measure of striatal dopamine (DA) activity (Jongkees \& Colzato, 2016). However, whether sEBR does indeed reflect DA activity is still debated today (Dang et al., 2017; Sescousse et al., 2018).

The connection between sEBR and DA first came from observations of neurological and psychiatric disorders that found decreased sEBR in patients with Parkinson's disease (Hall, 1945; Reddy, Patel, Hodge, \& Leavitt, 2013), a neurodegenerative disorder that affects the dopaminergic system in the brain, causing symptoms like rigidity (Dauer \& Przedborski, 2003). Schizophrenia has also been suggested to provide evidence for a connection between sEBR and DA due to 
excessive DA activity in the striatum (Howes, McCutcheon, \& Stone, 2015) and increased sEBR in schizophrenia patients (Adamson, 1995; Swarztrauber \& Fujikawa, 1998). Additionally, sEBR and DA have previously been investigated in pharmacological studies, which have observed an increase in sEBR after administration of DA agonists, while DA antagonists decreased sEBR in primates (Elsworth et al., 1991; Jutkiewicz \& Bergman, 2004). In one study, researchers found sEBR was correlated with dopamine levels specifically in the caudate nucleus in monkeys, suggesting that DA could regulate blink rate (Taylor et al., 1999). This is further supported by another study that found sEBR to be closely related to in vivo and positron emission tomography (PET) measures of striatal D2 receptor density in the ventral striatum and caudate nucleus of adult male vervet monkeys (Groman et al., 2014). These findings provide valuable evidence for sEBR being a viable measure of striatal DA activity and has led to many researchers to adopt sEBR as a measure of DA activity. Moreover, sEBR is an easy-torecord physiological measure that is non-invasive and affordable.

One cognitive process of interest, that is also closely related to DA activity, is working memory (WM) which is the process of actively holding information online and manipulating it to meet task demands (Baddeley, 1992). Prior research has found substantial evidence that demonstrates the importance of dopaminergic neurotransmission and the role of the prefrontal cortex during WM function (Cools \& Robbins, 2004; Courtney, Petit, Maisog, Ungerleider, \& Haxby, 1998; Funahashi, Bruce, \& Goldman-Rakic, 1989; Fuster \& Alexander, 1971; Wager \& Smith, 2003), especially during WM maintenance (Constantinidis et al., 2018; Funahashi et al., 1989; Fuster \& Alexander, 1971). Specifically, human studies investigating DA in WM tasks have found 
both caudate dopamine activity during WM maintenance and DA synthesis capacity to be positively correlated with WM capacity, a measure of the amount of information that can be held in WM (Cools, Gibbs, Miyakawa, Jagust, \& D'Esposito, 2008; Landau, Lal, O'Neil, Baker, \& Jagust, 2009). Though it is widely accepted that the PFC plays an important role in WM function (Roberts, Robbins, \& Weiskrantz, 1998), many researchers still debate the PFC's role in WM (Seamans \& Yang, 2004). One model that attempts to elucidate the PFC's role in WM function is the prefrontal cortex basal ganglia WM model (PBWM) (Frank, Loughry, \& O'Reilly, 2001; Hazy, Frank, \& O'Reilly, 2006). PBWN is a computational neural network model that suggests that WM requires robust maintenance and rapid selective updating. This model states that the frontal cortex facilitates robust, active maintenance through recurrent excitation in frontal neurons, while the basal ganglia orchestrates a gating mechanism that controls the flow of information into WM (Frank et al., 2001). Previous research has pointed towards DA being important for this sustained firing activity in the PFC during WM maintenance (de Frias et al., 2010; Durstewitz \& Seamans, 2008; Sawaguchi, 2001). The relationship between DA and WM performance is believed to follow an inverted-U-shape, in which too little or too much dopamine impairs performance, as seen in psychopharmacological studies (Stewart \& Plenz, 2006). In one study, the effects of administered dopaminergic drugs on PFC function depended on baseline levels of performance, whereas administration of bromocriptine, a dopamine agonist, impaired performance for individuals with higher working memory abilities while improving performance for individuals with lower working memory abilities (Kimberg, D'Esposito, \& Farah, 1997). 
Although sEBR has been used in prior research to investigate cognitive functions like WM, many of these studies relied on baseline levels of sEBR to investigate these relationships (Tharp \& Pickering, 2011; Unsworth, Robison, \& Miller, 2019; Zhang et al., 2015). Few studies have investigated the relationship between phasic sEBR during a WM task. Phasic sEBR refers to the measuring of sEBR in response to stimulus conditions while tonic sEBR refers to baseline levels of blinking (Bacher \& Allen, 2009). To the best of our knowledge, only one other study has examined sEBR as a function of different task phases (e.g., stimulus encoding, maintenance during the delay period, and stimulus probe periods) of a WM task (Bacher, Retz, Lindon, \& Bell, 2017). Bacher et al. (2017) found modulation of sEBR across these different phases are developed in infants as young as 10 months, indicating that $\mathrm{SEBR}$ can reflect dopamine function in early human development. They also observed higher sEBR during the Hide (delay) phase of the task in relation to the Reveal phase, which is when the experimenter revealed the toy's location to the child. This modulation of sEBR was suggested to reflect the engagement of cognitive resources that have become available during the Hide phase and transiently elevated DA activity that is needed to update and maintain mental representations (Bacher et al., 2017).

The goal of the current study was to investigate how fluctuations in sEBR during different phases of a Sternberg visual WM task (Figure 1) relate to performance, and how sEBR fluctuations change across task demands. First, we hypothesized that sEBR during the WM Delay period, when stimuli are being maintained, would be positively correlated with task performance and that there would be a non-linear relationship such that low and high sEBR would correlate with worse performance. Second, we 
hypothesized differences in SEBR across phases of the WM task with differences between phasic sEBR during the WM delay and tonic sEBR during non-task rest periods.

\section{Material and methods}

\subsection{Participants}

The experiments were conducted under a protocol approved by the Institutional Review Board of The City University of New York Human Research Protection Program (CUNY HRPP IRB). All methods were carried out in accordance with the relevant guidelines and regulations of the CUNY HRPP IRB committee. All participants were recruited either by flyers posted throughout the City College of New York campus or by web postings on the City College of New York SONA online experimental scheduling system. All participants had normal or corrected-to-normal vision with no reported neurological or psychiatric disorders. Participants were either compensated $\$ 15$ per hour or received one psychology course credit per hour of participation in the study. Written informed consent was obtained from all participants in the study.

Participants selected for Experiment 1 and Experiment 2 were part of a larger study. Nineteen healthy participants (8 males; $M=23.79$; S.D. $=7.72$ ) were recruited for Experiment 1. In Experiment 1, sEBR was measured inside a 3 tesla Siemens Prisma MRI scanner. In Experiment 2, sEBR was recorded in a sound attenuated EEG booth during acquisition of EEG data while participants sat upright. Fifty-three healthy participants (29 males; $M=23.58 ;$ S.D. $=5.79)$ were recruited for Experiment 2. Six participants were removed from Experiment 1 including four participants who were 
removed for noisy data and two who were removed for task performance below or close to chance. A total of 19 participants were removed from Experiment 2 for multiple reasons including 11 participants who were removed due to bad EOG channel quality, four participants who were removed because of a stimulus marker malfunction, three participants who were removed due to outlier detection, and two who were removed for failing to adhere to the protocol. The final sample for the analysis in Experiment 1 was 13 subjects, and in Experiment 2 it was 34 subjects.

\subsection{Task and Procedure}

Prior to the start of the task, participants completed a 5-minute Rest period which consisted of staring at a black fixation cross that was shown on a grey background. Participants completed another 5-minute Rest period after completing three runs of the task. This fixation cross was also used during the delay period of the task. Participants completed three runs with each run containing 54 trials of a modified version of the Sternberg WM task (Sternberg, 1966). Naturalistic scenes were used as stimuli and were sampled from the SUN database (Xiao, Hays, Ehinger, Oliva, \& Torralba, 2010). The task consisted of a stimulus encoding period, delay period, probe period, and postprobe scrambled stimulus period (which served as a visual baseline and to signal end of trial). During the encoding period, participants were shown three subsequent novel scenes for $1400 \mathrm{~ms}$ each. During the delay period, a black fixation cross was shown on a grey background for varied lengths (either 2, 5, or 9 seconds long). The delay period duration was randomized from trial to trial to engage subjects' attention consistently across trials because they could not predict when the delay period would end. Each of the three task runs had 18 trials of each delay duration with the order randomized. The 
probe was presented for $1400 \mathrm{~ms}$ after the delay period and consisted of a new image (one that had not been presented yet) or an old image (one that was shown during encoding). The chance of receiving a new probe was $50 \%$. Participants indicated whether the image presented was either a new or an old image with a button press. After the probe, a Fourier phase-scrambled scene was shown for 2000 ms, indicating the end of the current trial followed by a jittered period of blank screen before the next trial began.

\section{3 sEBR Recording}

Participants were not given instructions about when to blink during the experiment. Previous studies have found blink rate to be stable between 10 am and 5 pm (Barbato et al., 2000; Doughty \& Naase, 2006). For both Experiment 1 and Experiment 2, sEBR was recorded between 10:00 am and 3:00 pm. During Experiment 1, eye blinks were recorded inside a 3 tesla Siemens Prisma MRI scanner using an MRI compatible EyeLink 1000 Eye Tracker (SR Research) and was recorded at $500 \mathrm{~Hz}$. In Experiment 2, eye blinks were recorded using an electrooculogram (EOG) that was recorded during 64-channel scalp electroencephalogram (EEG) using a Brain Products cap and active electrode recording system (actiChamp). EOGs were placed above the left eye and below the right eye to track blinking. Blink detection was performed using MNE Python via the function "find_eog_events" (Gramfort et al., 2013). Blink epochs were evaluated for each run of the task for all participants. Runs with blink epochs which did not resemble the standard blink shape were removed from the analysis. Only participants with 2 or more runs of good eye-tracking data were used in the analysis. The first two seconds of all delay periods were used in the analysis. In Experiment 1 and 2, sEBR 
was computed by dividing the total number of blinks by the total period duration for any given phase resulting in units of blinks per minute:

$$
s E B R=\frac{\text { total blinks }}{\text { total period duration }}
$$

\subsection{Statistical analysis}

The statistical results reported in this study were computed using JASP (Version 0.14.1). We investigated the relationship between sEBR and WM task accuracy using Spearman's rho. Spearman's rho was used to measure the relationship between sEBR and WM performance due to previous reports that DA follows an "inverted U-shape" with WM performance. We hypothesized that the relationship between sEBR and WM performance would represent a monotonic function, and thus predicted Spearman's rho to be the more appropriate measure. sEBR and task accuaracy data were checked for outliers prior to analysis and were removed. Finally, we investigated the non-linear relationship between sEBR during the Delay period of the task, in both Experiment 1 and Experiment 2, and task accuracy by computing a $2^{\text {nd }}$ order polynomial regression model.

\section{Results}

\subsection{Experiment 1}

In Experiment 1, we examined the relationship between sEBR and WM task performance while the duration of the WM delay period interval was varied. The first two seconds of all Delay periods were used in the analysis. First, because of the previously reported non-linear relationship between DA and WM task performance (Cools \& D'Esposito, 2011), Spearman's rho correlation coefficient $\left(r_{\mathrm{s}}\right)$ was used to analyze the 
relationship between sEBR and task accuracy. After performing Bonferroni multiple comparisons correction on p-values, we found no significant relationship between sEBR during the phases of the task and task accuracy (Figure 2a). However, there was a marginally significant positive correlation between sEBR during the Delay period and task accuracy $\left(r_{\mathrm{s}}=.634, p=.020\right)$ (Figure $\left.2 \mathrm{a}\right)$. We then examined the correlation between sEBR during the whole trial period and task accuracy to make sure that this relationship was not driving the relationship between sEBR during the Delay and task accuracy. There was no significant relationship between sEBR during the whole trial and task accuracy $\left(r_{\mathrm{s}}=.146, p=.634\right)$ (Figure $\left.2 \mathrm{~b}\right)$. Second, we computed a one-way ANOVA test to compare participants' sEBR across the task phases. A Levene's test of variance was first computed to check the homogeneity of variance; variance was not violated ( $p=.087$ ). Normality was then checked using a $Q-Q$ plot. No deviations were noted. There were significant differences in sEBR among the group means $(F(3,48)=$ 14.071, $p<.001)$. A Tukey post-hoc test revealed that sEBR was significantly lower during Encoding (18.3 \pm 11.9 sEBR, $p=.001)$ and Probe $(10.5 \pm 6.7$ sEBR, $p<.001)$ periods compared to the Delay period (39.85 \pm 19.3 sEBR) (Figure 3$)$. There was no significant difference in sEBR between the Delay and Scrambled period $(p=.981)$. sEBR was also significantly lower during Encoding (18.3 \pm 11.9 sEBR, $p=.004)$ and Probe $(10.5 \pm 6.7$ sEBR, $p<.001)$ periods compared to the Scrambled period $(37.7 \pm$ 14.6 sEBR) (Figure 3). Finally, we investigated the difference between phasic sEBR during the Delay period and tonic sEBR during the Rest period. We performed a paired samples t-test to compare sEBR during the Delay and during Rest. We observed sEBR to be significantly higher during the Delay period (39.9 $\pm 19.3 \mathrm{sEBR})$ compared to the 
Rest period $(25.9 \pm 13.9 \mathrm{sEBR}), \mathrm{t}_{(13)}=3.544, \mathrm{p}=.004$ (Figure 4a). We then investigated the correlation between tonic sEBR during the Rest period and task accuracy. There was no significant correlation between sEBR during the Rest period and task accuracy $\left(r_{\mathrm{s}}=.305, p=.311\right)($ Figure $4 b)$.

\subsection{Experiment 2}

Experiment 2 included a larger sample of subjects with a task design identical to Experiment 1. First, we examined the relationship between sEBR during each WM task phase and task accuracy. After performing Bonferroni correction on p-values, we found that sEBR during the WM delay period was significantly positively correlated with task performance $\left(r_{\mathrm{s}}=.508, p=.002\right)$, with no significant relationships observed between sEBR in other task periods and task performance (Figure 5a). We then examined the relationship between sEBR during the whole trial and task accuracy to make sure that the significant relationship between Delay sEBR and task accuracy was not driven by sEBR during the whole trial. We found no significant relationship between whole trial sEBR and task accuracy $\left(r_{\mathrm{s}}=.192, p=.278\right)$ (Figure $\left.5 \mathrm{~b}\right)$. We then repeated the same analysis of comparing sEBR across the task phases by computing a one-way ANOVA test. A Levene's test of variance was computed and was found to be significant $(p<$ .001). Therefore, a Welch's ANOVA was performed. Normality was then checked using a $Q-Q$ plot. No deviations were noted. There were significant differences in sEBR among the group means $(\mathrm{F}(3,65.8)=35.731, p<.001)$. A Games-Howell Tukey posthoc test revealed that sEBR was significantly lower during Encoding $(11.6 \pm 8.0 \mathrm{sEBR}$, $p<.001)$, Probe $(7.3 \pm 4.1$ sEBR, $p<.001)$, and Scrambled $(19.5 \pm 12.6$ sEBR, $p<$ $.001)$ periods compared to the Delay period (35.6 $\pm 18.3 \mathrm{sEBR}$ ) (Figure 6). sEBR was 
also significantly lower during the Encoding (11.6 \pm 8.0 sEBR, $p=.005)$ and Probe (7.3 \pm 4.1 sEBR, $p<.001)$, periods compared to the Scrambled period $(19.5 \pm 12.6 \mathrm{sEBR})$ (Figure 6). Additionally, sEBR was significantly lower during the Probe $(7.3 \pm 4.1$ sEBR, $p=.034)$, period compared to the Encoding period (11.6 $\pm 8.0 \mathrm{sEBR}$ ) (Figure 6). We then investigated the difference between sEBR during the Delay period and sEBR during the Rest period. We performed a paired samples t-test to compare sEBR during the Delay and during Rest. We observed sEBR to be significantly higher during the Delay period (35.6 $\pm 18.3 \mathrm{sEBR})$ compared to the Rest period $(17.7 \pm 11.1 \mathrm{sEBR}), \mathrm{t}_{(33)}=$ $6.005, p<.001$ (Figure 7a). We then investigated the correlation between tonic sEBR during the Rest period and task accuracy. There was no significant correlation between sEBR during the Rest period and task performance $\left(r_{\mathrm{s}}=-0.053, p=.768\right)$ (Figure 6b).

\subsection{Polynomial Regression Model}

To investigate whether sEBR during the Delay varies non-linearly with task performance, we computed a quadratic polynomial regression model between sEBR during the Delay period of Experiment 1 and Experiment 2 and task accuracy. Although the non-linear fits explained approximately $20 \%$ of the variance in each experiment, there was no significant $2^{\text {nd }}$ order relationship found between task accuracy and sEBR during the first two seconds of the Delay in Experiment $1(\beta=-0.625, p=0.638)$ nor between task accuracy and sEBR during the first two seconds of the Delay in Experiment $2(\beta=-0.568, p=0.322)$ (Figure 8).

\section{Discussion}


In the present study, we investigated the temporal fluctuations in sEBR across a WM paradigm and its relation to WM task accuracy in two experiments, inside and outside an MRI scanner, and using two methods of measuring sEBR. Using the same Sternberg working memory paradigm, we observed a positive relationship between sEBR and task performance only during the WM task delay in both experiments. We also found a significant difference in sEBR between task phases and a difference between Delay period sEBR and baseline sEBR.

Our first hypothesis was that phasic sEBR during the Delay period of the WM task would be positively correlated with task accuracy and that we would also observe a non-linear relationship where high and low sEBR would be predictive of low performance. We observed a positive correlation between sEBR during the Delay period in both Experiment 1 and Experiment 2 with task accuracy. However, only in Experiment 2 did this relationship survive stringent multiple comparisons corrections to reach significance. We believe that the lack of significance in Experiment 1 is due to statistical power limitations due to the smaller sample size. If we interpret sEBR as an indirect measure of striatal DA activity, as other studies have postulated, we could speculate that higher sEBR during the WM delay is correlated with task accuracy due to DA regulating the maintenance and updating of representations in WM (Westbrook \& Braver, 2016). The other results support this idea since no other task period was significantly correlated with task accuracy. Many studies that have investigated the relationship between sEBR and cognitive functions have used baseline levels of sEBR taken before or after tasks in their analysis (Tharp \& Pickering, 2011; Unsworth, Robison, et al., 2019; Zhang et al., 2015). However, we show with the present results 
that while the WM task Delay period sEBR was correlated positively with task accuracy, baseline levels of sEBR were not. Our results highlight the importance of examining phasic and tonic sEBR when investigating the relationships between sEBR and other cognitive functions. The results also highlight the importance of not restricting participants from blinking during the WM delay as the rate of blinking could provide insight into processes related to WM maintenance. We also investigated the proposed “Inverted-U-shape" relationship between DA and WM performance by computing a $2^{\text {nd }}$ order polynomial regression model between sEBR during the delay and task accuracy (Cools \& D'Esposito, 2011). Though the non-linear model showed explained approximately $20 \%$ of the variance, the fit was not significant. We believe that failure to achieve non-linear model significance was due to lack of extreme (sub- and supraoptimal) sEBRs observed in the pool of participants, which are typically found in clinical populations (e.g., with Schizophrenia) (Adamson, 1995; Swarztrauber \& Fujikawa, 1998). Future studies should investigate sEBR with healthy subjects and with subjects that have been observed to have extreme sEBR in order to have a wider variety of sEBRs and to better understand its connection with DA. Additionally, other methods of DA measures could be used to investigate DA during the delay period such as correlations with neuromelanin-sensitive MRI which can detect neuromelanin, a product of dopamine metabolism (Cassidy et al., 2019).

Our second hypothesis was that we would see significant differences in sEBR across the WM task as well as between sEBR during Rest and during the Delay period. We found sEBR to be the lowest during periods like Encoding and Probe in both Experiments, while sEBR during the Delay was the highest. Our results support 
previous findings which found task-related modulation of sEBR (Oh et al., 2012; Siegle et al., 2008). Prior work has found sEBR to be lower during tasks that require visual attention (Fukuda, Stern, Brown, \& Russo, 2005; Oh et al., 2012). This would explain the lower sEBR's observed during the Encoding period when participants are encoding information into WM and during the Probe period where participants are retrieving information from WM. We also found that sEBR was the highest during the Delay period when participants were maintaining information in WM. This was also demonstrated in a different study which investigated sEBR during an A-not-B WM task where infants had to search for a hidden toy by making an eye movement to one of two locations (Bacher et al., 2017). Higher sEBR during the WM delay could be due to DA regulating the maintenance and updating of representations in WM (Westbrook \& Braver, 2016), but this remains speculation until further studies directly measure dopaminergic activity during task performance. Our results further support this interpretation since Delay period sEBR was significantly higher than baseline sEBR during the Rest period. Lower sEBR during the Rest period could be due to the lack of need to update or maintain WM during this period.

To conclude, we investigated temporal changes of sEBR during different phases of a WM task and its relation to WM performance. We observed a positive correlation between sEBR and WM task performance during the Delay period, but not during the other phases of the task. Additionally, we found evidence for an association of sEBR during both stimulus encoding and WM probe retrieval, potential reflecting demands on visual attention. To the best of our knowledge, this is the first study to investigate phasic and tonic sEBR during different phases of a WM task using complex visual scenes. 
Future studies should continue to investigate sEBRs in relation to direct measures of cortical (especially PFC) and subcortical dopamine and assess linear and non-linear relationships with task performance in healthy and clinical populations including Schizophrenia and Parkinson's disease.

\section{Figures}
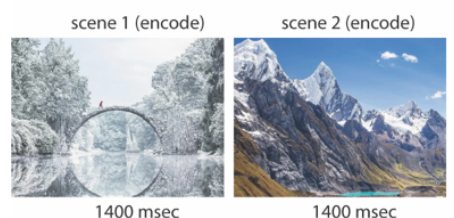

$1400 \mathrm{msec}$

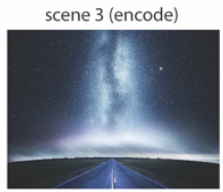

$1400 \mathrm{msec}$

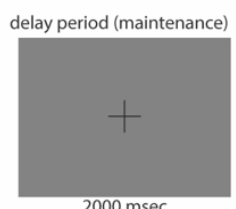

$2000 \mathrm{msec}$

$5000 \mathrm{msec}$

or
$9000 \mathrm{msec}$

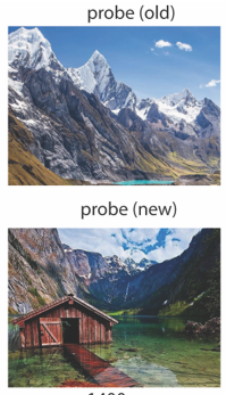

$1400 \mathrm{msec}$

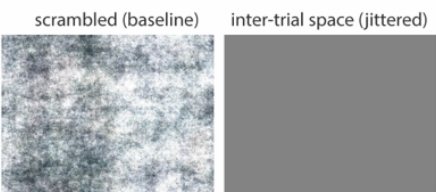

$2000 \mathrm{msec}$

Figure 1. Task design. Each trial began with an encoding period in which three novel complex scenes were presented for $1400 \mathrm{~ms}$ each. The encoding period was followed by a delay period where a fixation cross was presented on a grey background for a varied amount of time (2 s, $5 \mathrm{~s}$, or $9 \mathrm{~s})$. After the delay period, the probe was presented for 1400 ms and participants had to identify whether with a button press whether the image was a new image or one of the previously presented images. After the probe, a scrambled image was presented for $2000 \mathrm{~ms}$ which indicated the end of the trial followed by jittered blank space before the start of the next trial. 
a)

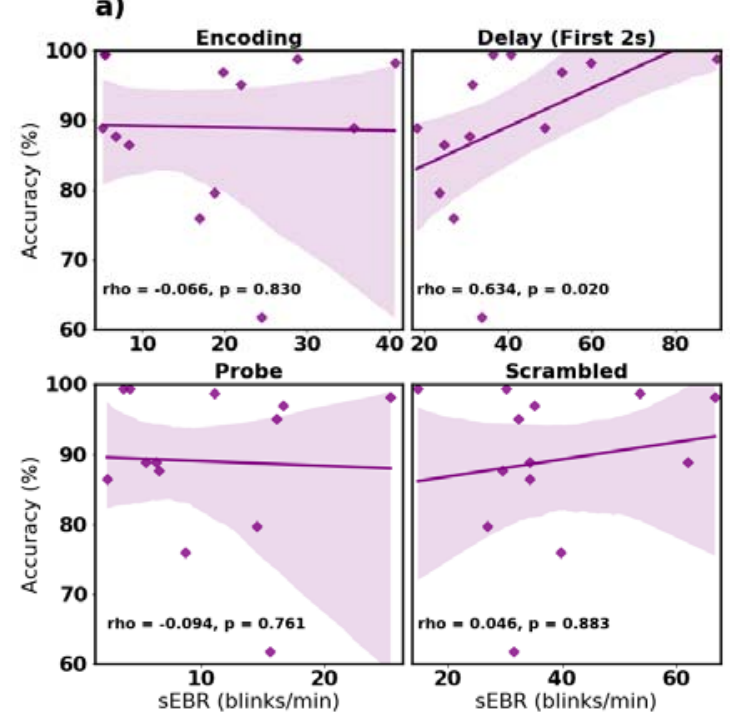

b)

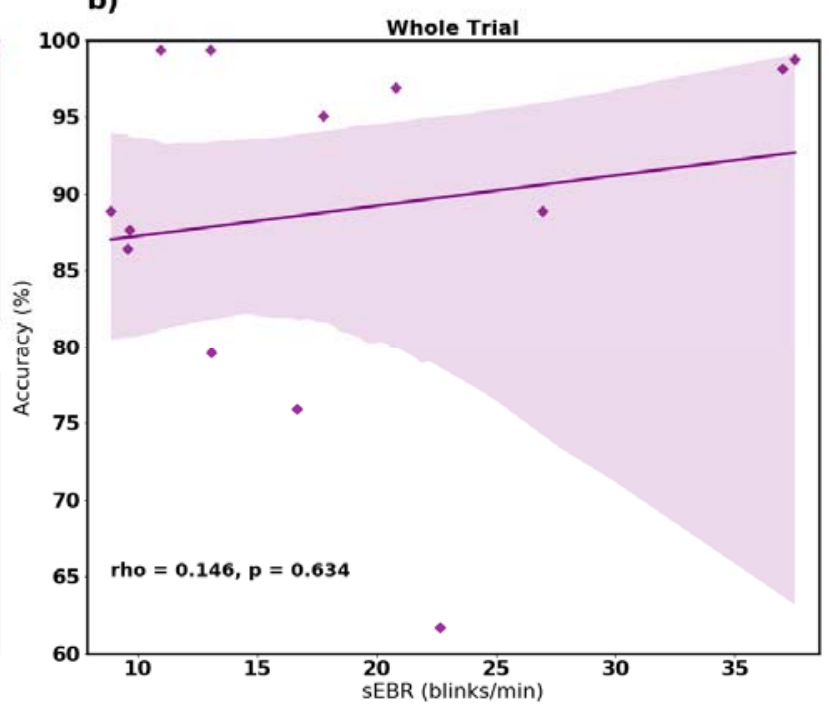

Figure 2. Correlation between SEBR during different phases of the task and task accuracy in Experiment 1. Correlation plots show sEBR (in blinks/min) on the x-axis and task accuracy (\% correct) on the y-axis. a) These four plots show encoding (top left), the first two seconds of the delay (top right), probe (bottom left), and scrambled (bottom right) periods. The delay period shows a positive correlation $(p=0.02$, but not significant after multiple comparisons correction) between task accuracy and sEBR during the first two seconds of the delay period. b) This plot represents the relationship between SEBR during the whole trial and task accuracy. Fitted line represents linear regression model fit. Shaded region depicts $95 \%$ confidence interval. Note: $p$-values for Figure 2a. after Bonferroni multiple comparison correction: ${ }^{*} p<.0125,{ }^{* *} p<.0025{ }^{* * *}$ $p<.00025$. $p$-values for figure $2 b .{ }^{*} p<.05,{ }^{* *} p<.01,{ }^{* * *} p<.001$. 


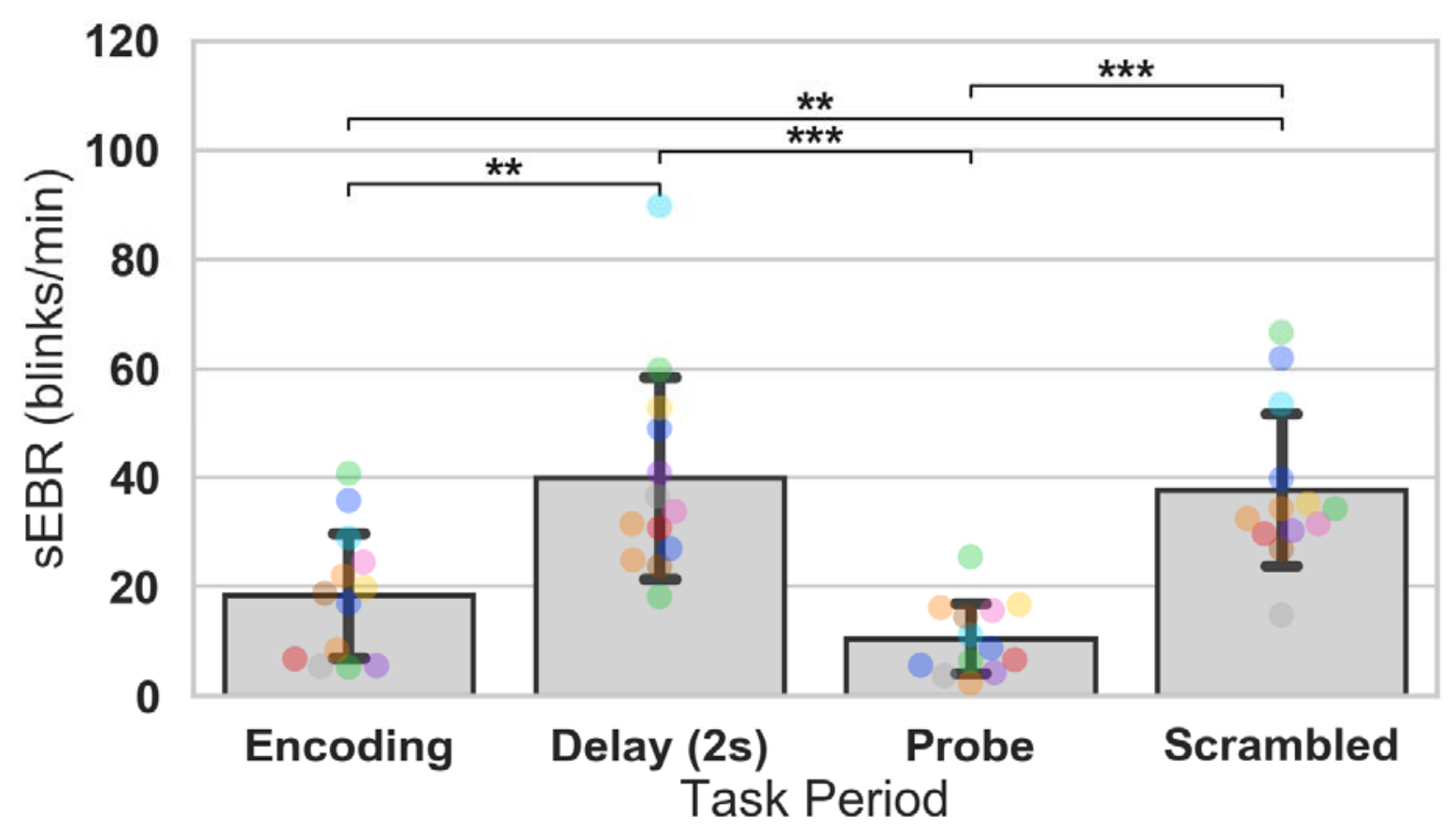

Figure 3. ANOVA test of sEBR across task periods in Experiment 1. Bar plots show task period on the x-axis and sEBR (blinks/min) on the $y$-axis. Delay period sEBR was significantly greater than Encoding and Probe sEBR. Scrambled sEBR was also significantly greater than Encoding and Probe sEBR. Error bars depict 95\% confidence interval. Note: Each colored circle represents an individual participant; some colors may be presented twice in one bar due to limited primary colors available for display. Pvalues were adjusted for comparing a family of $4 .{ }^{*} p<.05,{ }^{* *} p<.01,{ }^{* * *} p<.001$. 

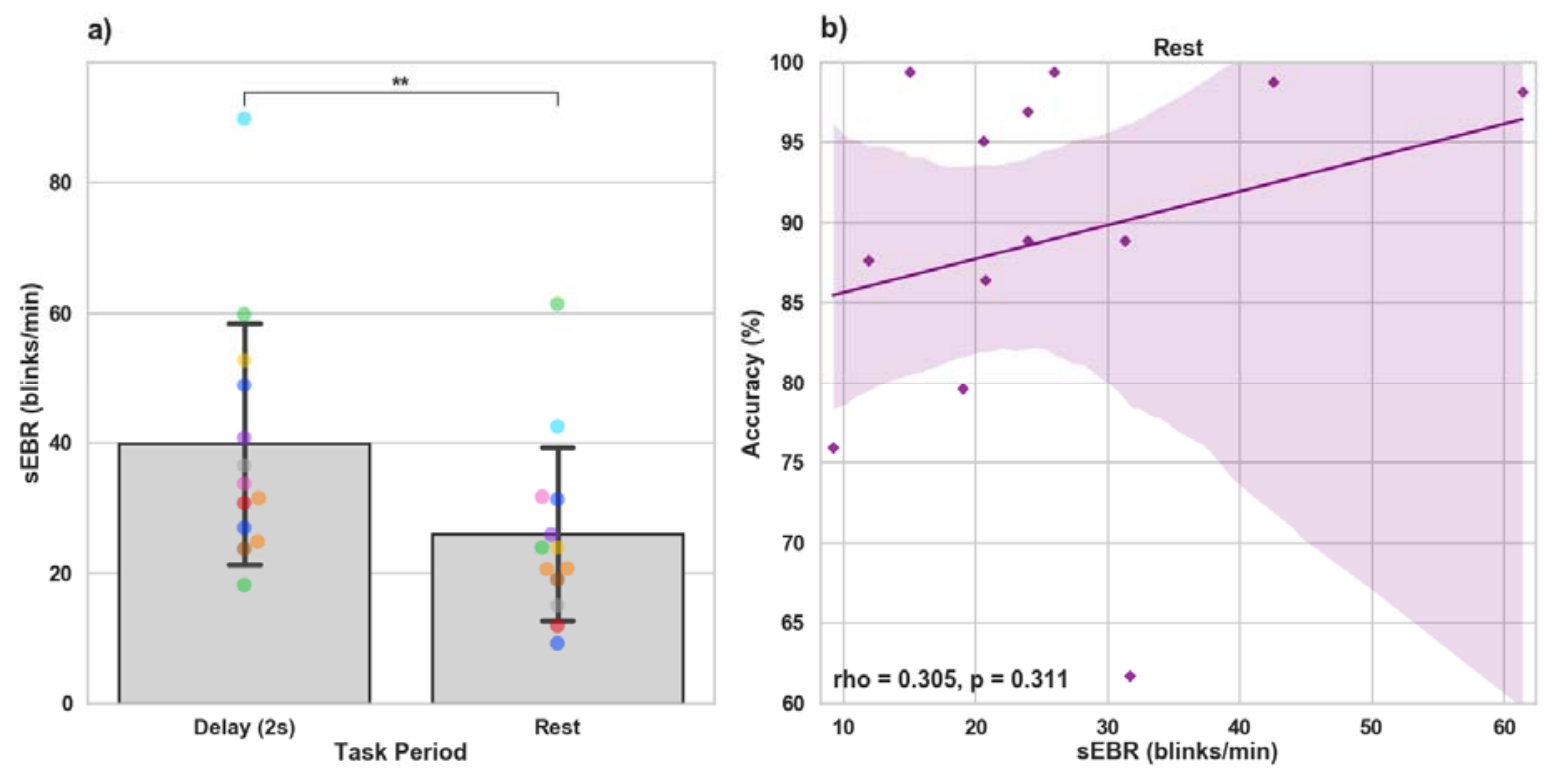

Figure 4. Paired T-tests between Delay period sEBR and Rest sEBR and correlation between Rest sEBR and task accuracy in Experiment 1. a) Bar plots show task period on the $x$-axis and sEBR on the y-axis. Delay period sEBR was significantly higher than Rest sEBR. Error bars depict 95\% confidence interval. b) Correlation plot of sEBR during the Rest period on the x-axis and task accuracy on the y-axis. Fitted line represents linear regression model fit. Shaded region depicts $95 \%$ confidence interval. ${ }^{*} p<.05,{ }^{* *} p<.01,{ }^{* * *} p<.001$. 
a)

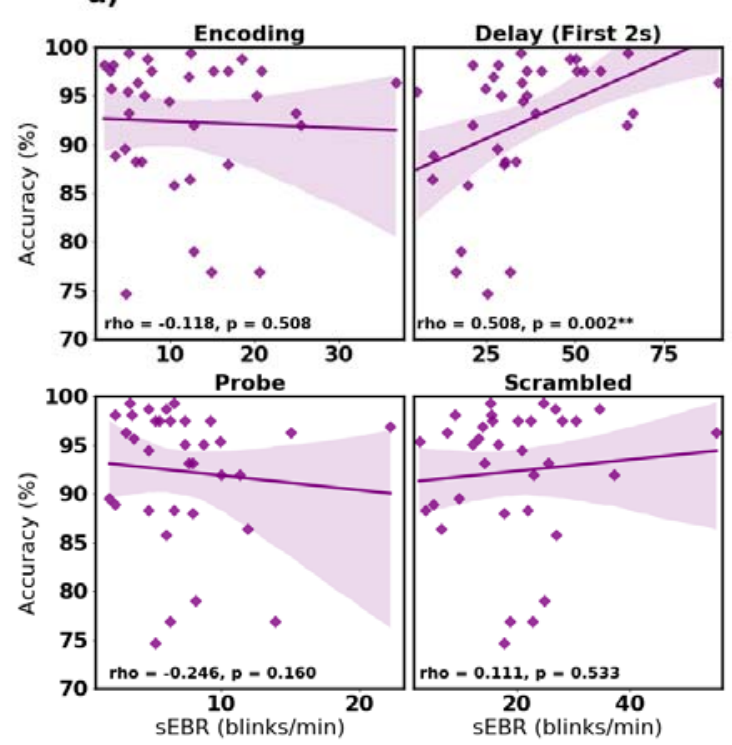

b)

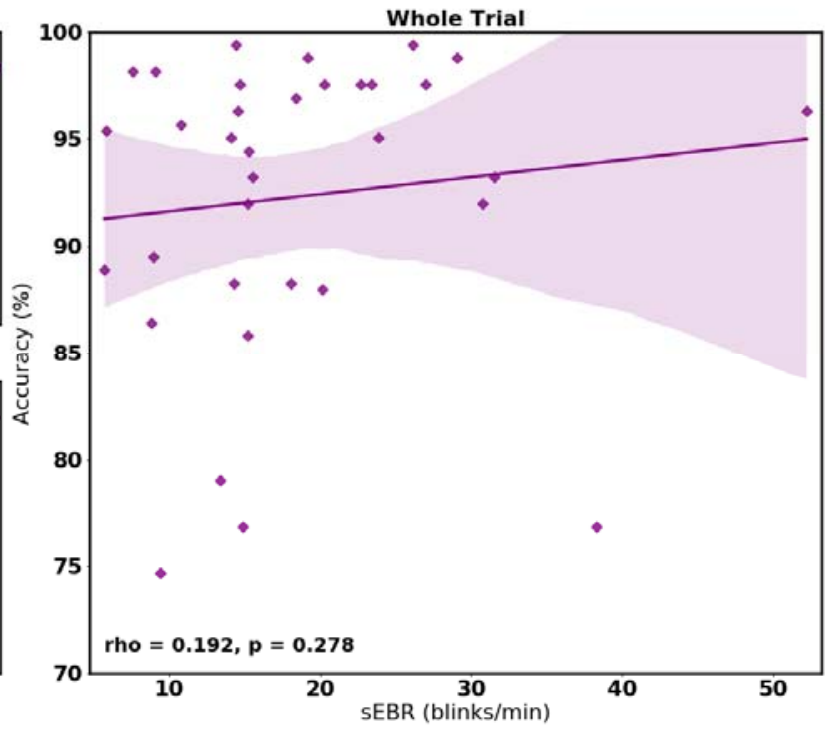

Figure 5. Correlation between sEBR during different phases of the task and task accuracy in Experiment 2. Correlation plots show sEBR on the x-axis and task accuracy on the y-axis. a) These four plots are encoding (top left), the first two seconds of the delay (top right), probe (bottom left), and scrambled (bottom right) periods. The delay period shows a strong positive correlation $(p=0.002$, which was significant after a multiple comparison correction) between task accuracy and sEBR during the first two seconds of the delay period. b) This plot represents the relationship between sEBR during the whole trial and task accuracy. Fitted line represents linear regression model fit. Shaded region depicts $95 \%$ confidence interval. Note: $p$-values for figure $5 \mathrm{a}$. after Bonferroni correction: ${ }^{*} p<.0125,{ }^{* *} p<.0025{ }^{* *} p<.00025$. $p$-values for figure $5 b .{ }^{*} p$ $<.05,{ }^{* *} p<.01,{ }^{* * *} p<.001$. 


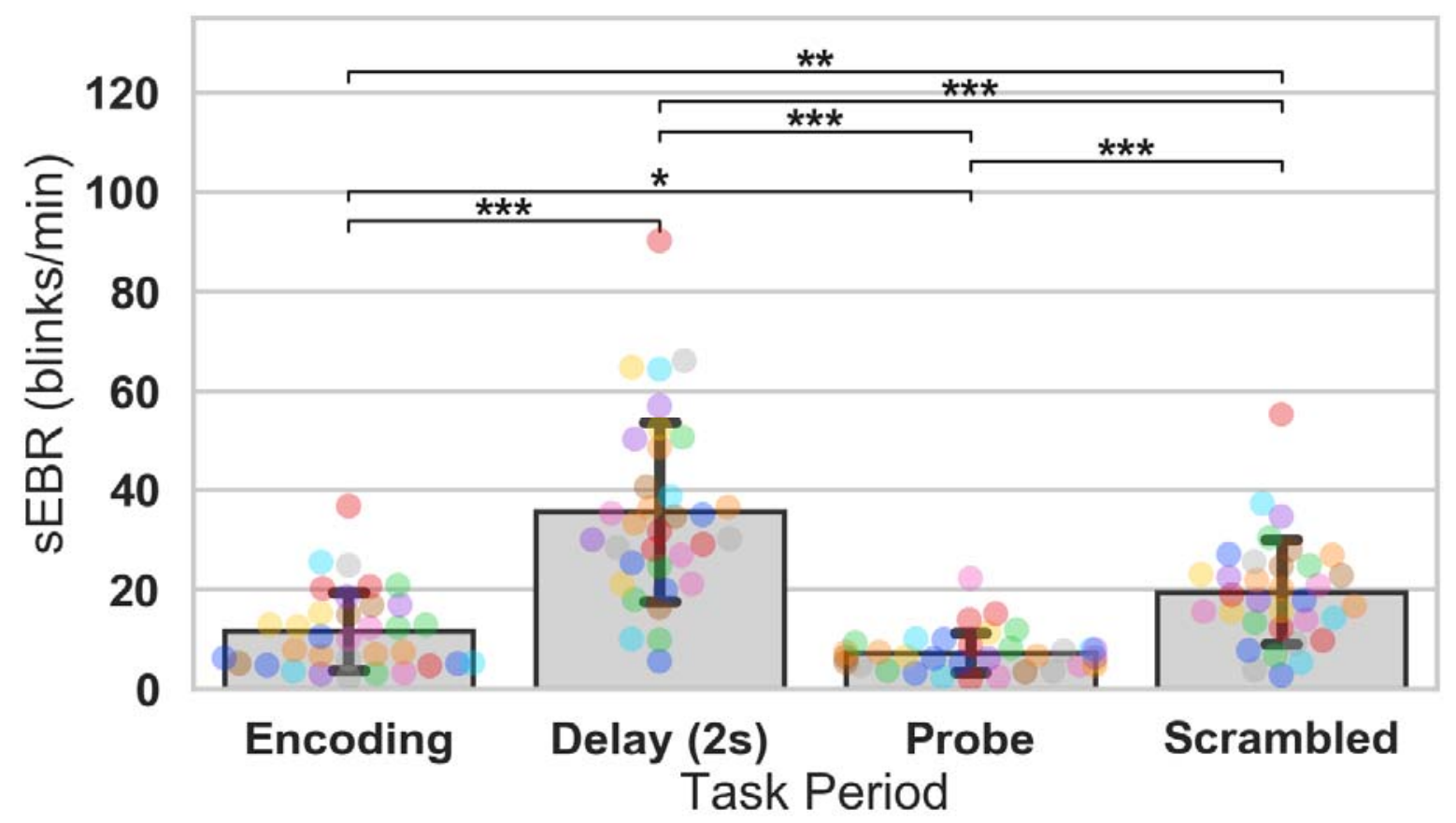

Figure 6. ANOVA test of sEBR across task periods in Experiment 2. Bar plots show task period on the x-axis and sEBR on the y-axis. Delay period sEBR was significantly greater than Encoding, Probe, and Scrambled sEBR. Scrambled sEBR was also significantly greater than Encoding and Probe sEBR. Encoding sEBR was significantly greater than Probe sEBR. Error bars depict 95\% confidence interval. Note: Each colored circle represents an individual participant; some colors may be presented twice in one bar due to limited primary colors available for display. P-values were adjusted for comparing a family of $4 .{ }^{*} p<.05,{ }^{* *} p<.01,{ }^{* * *} p<.001$. 

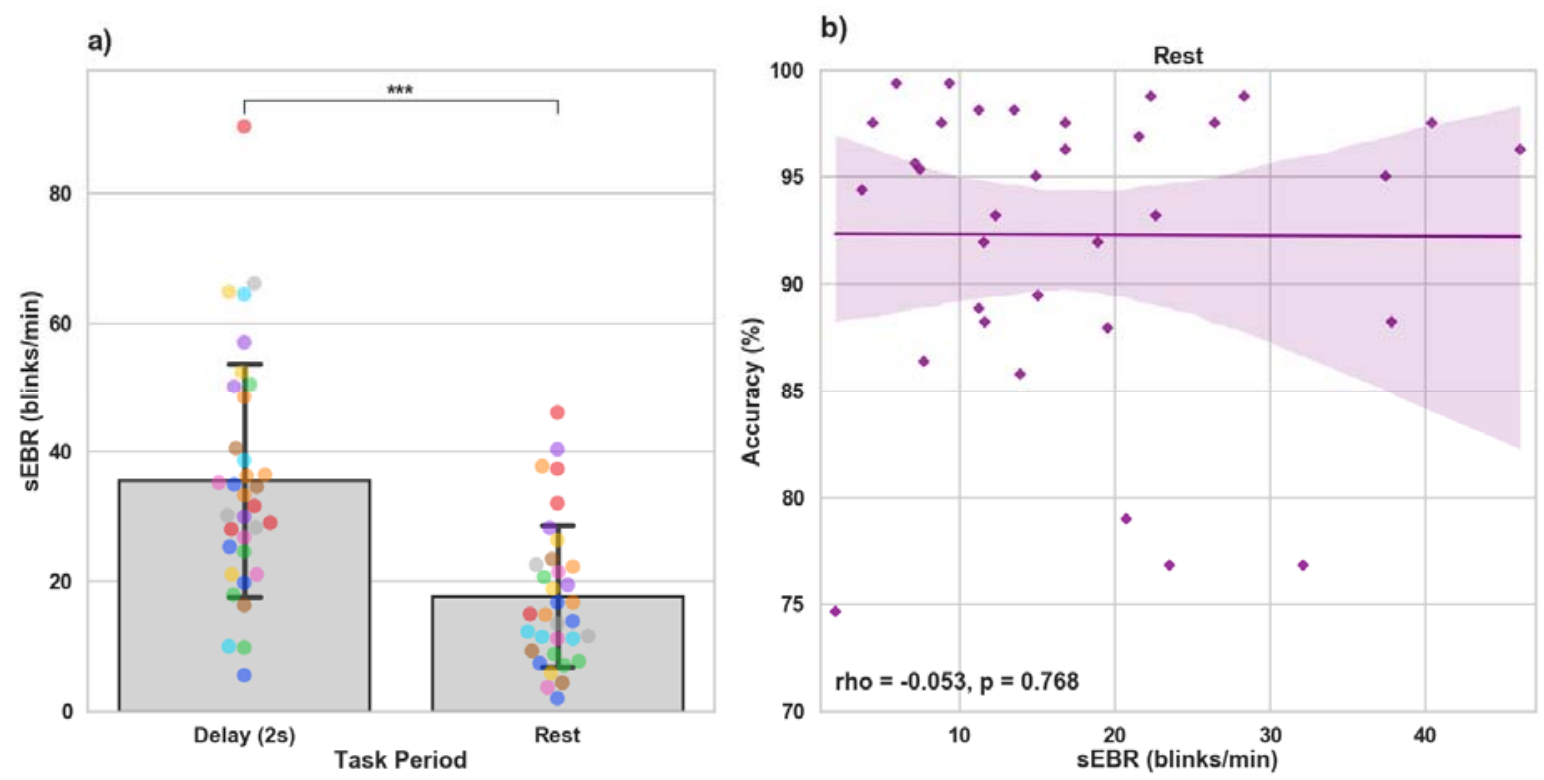

Figure 7. Paired T-tests between Delay period sEBR and Rest sEBR and correlation between Rest sEBR and task accuracy in Experiment 2. a) Bar plots show task period on the $x$-axis and sEBR on the y-axis. Delay period sEBR was significantly higher than Rest sEBR. Error bars depict 95\% confidence interval. b) Correlation plot of sEBR during the Rest period on the x-axis and task accuracy on the y-axis. Fitted line represents linear regression model fit. Shaded region depicts $95 \%$ confidence interval. ${ }^{*} p<.05,{ }^{* *} p<.01,{ }^{* * *} p<.001$. 
a)

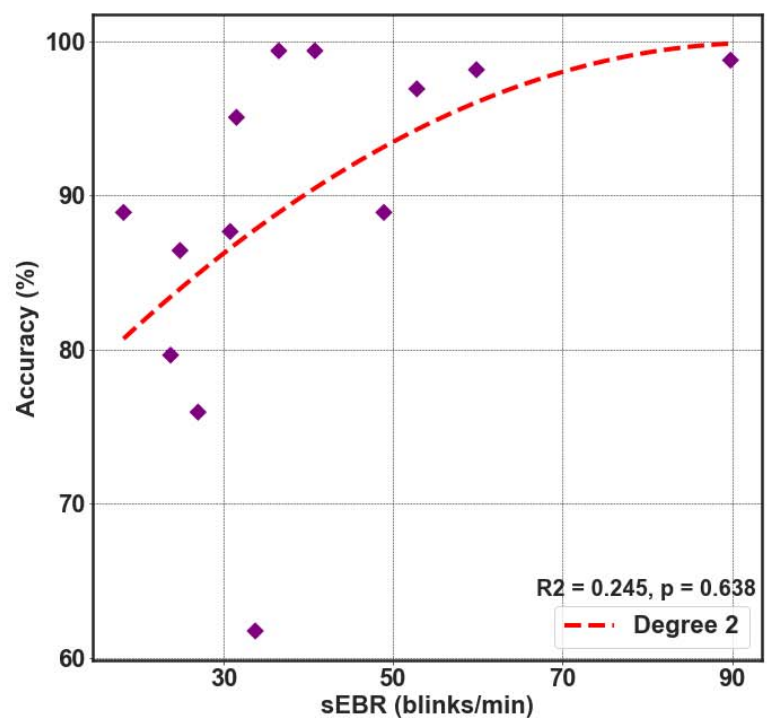

b)

Experiment 2 (Delay 2s)

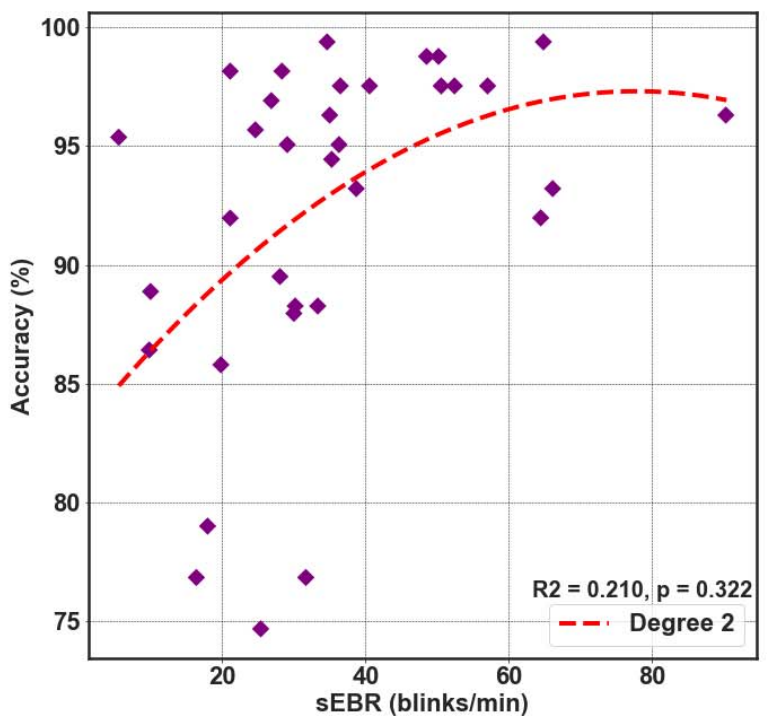

Figure 8. Polynomial regression model between task accuracy and sEBR during the first two seconds of the delay for Experiment 1 and Experiment 2. Regression plots show sEBR during the first two seconds of the Delay on the $x$-axis and task accuracy on the $y$-axis. a) $2^{\text {nd }}$ order polynomial regression model fitted on sEBR during the Delay and task accuracy in Experiment 1. b) $2^{\text {nd }}$ order polynomial regression model fitted on sEBR during the Delay and task accuracy in Experiment 2. The fitted red line represents $2^{\text {nd }}$ order polynomial regression model fit. The relationship between sEBR and WM performance appears to be non-linear and explains about $20 \%$ of the variance in each experiment but neither fit reached significance. ${ }^{*} p<.05,{ }^{* *} p<.01,{ }^{* * *} p<.001$. 


\section{Acknowledgments}

Research reported in this publication was supported by the National Institute of Mental

Health of the National Institutes of Health under Award Number R56MH116007 (T.M.E.).

\section{Author contributions}

T.M.E designed the study. J.O., C.R, and B.G performed the research. J.O. analyzed and interpreted the data, prepared the figures, and wrote the final manuscript. C.R, B.G, and T.M.E edited and reviewed the manuscript.

\section{Competing interests}

The authors declare no competing interests. 


\section{References}

Adamson, T. A. (1995). Changes in blink rates of Nigerian schizophrenics treated with chlorpromazine. West Afr J Med, 14(4), 194-197.

Bacher, L. F., \& Allen, K. J. (2009). Sensitivity of the rate of spontaneous eye blinking to type of stimuli in young infants. Dev Psychobiol, 51(2), 186-197.

Bacher, L. F., Retz, S., Lindon, C., \& Bell, M. A. (2017). Intraindividual and Interindividual Differences in Spontaneous Eye Blinking: Relationships to Working Memory Performance and Frontal EEG Asymmetry. Infancy, 22(2), 150-170.

Baddeley, A. (1992). Working Memory. Science, 255(5044), 556-559.

Barbato, G., Ficca, G., Muscettola, G., Fichele, M., Beatrice, M., \& Rinaldi, F. (2000). Diurnal variation in spontaneous eye-blink rate. Psychiatry Res, 93(2), 145-151.

Bentivoglio, A. R., Bressman, S. B., Cassetta, E., Carretta, D., Tonali, P., \& Albanese, A. (1997). Analysis of blink rate patterns in normal subjects. Movement Disorders, 12(6), 10281034.

Cassidy, C. M., Zucca, F. A., Girgis, R. R., Baker, S. C., Weinstein, J. J., Sharp, M. E., et al. (2019). Neuromelanin-sensitive MRI as a noninvasive proxy measure of dopamine function in the human brain. Proceedings of the National Academy of Sciences of the United States of America, 116(11), 5108-5117.

Colzato, L. S., van den Wildenberg, P. M., van Wouwe, N. C., Pannebakker, M. M., \& Hommel, B. (2009). Dopamine and inhibitory action control: evidence from spontaneous eye blink rates. Experimental Brain Research, 196(3), 467-474.

Constantinidis, C., Funahashi, S., Lee, D., Murray, J. D., Qi, X. L., Wang, M., et al. (2018). Persistent Spiking Activity Underlies Working Memory. J Neurosci, 38(32), 7020-7028.

Cools, R., \& D'Esposito, M. (2011). Inverted-U-Shaped Dopamine Actions on Human Working Memory and Cognitive Control. Biological Psychiatry, 69(12), E113-E125.

Cools, R., Gibbs, S. E., Miyakawa, A., Jagust, W., \& D'Esposito, M. (2008). Working memory capacity predicts dopamine synthesis capacity in the human striatum. Journal of Neuroscience, 28(5), 1208-1212.

Cools, R., \& Robbins, T. W. (2004). Chemistry of the adaptive mind. Philosophical Transactions of the Royal Society a-Mathematical Physical and Engineering Sciences, 362(1825), 28712888.

Courtney, S. M., Petit, L., Maisog, J. M., Ungerleider, L. G., \& Haxby, J. V. (1998). An area specialized for spatial working memory in human frontal cortex. Science, 279(5355), 1347-1351.

Dang, L. C., Samanez-Larkin, G. R., Castrellon, J. J., Perkins, S. F., Cowan, R. L., Newhouse, P. A., et al. (2017). Spontaneous Eye Blink Rate (EBR) Is Uncorrelated with Dopamine D2 Receptor Availability and Unmodulated by Dopamine Agonism in Healthy Adults. Eneuro, 4(5). 
Dauer, W., \& Przedborski, S. (2003). Parkinson's disease: Mechanisms and models. Neuron, 39(6), 889-909.

de Frias, C. M., Marklund, P., Eriksson, E., Larsson, A., Oman, L., Annerbrink, K., et al. (2010). Influence of COMT Gene Polymorphism on fMRI-assessed Sustained and Transient Activity during a Working Memory Task. Journal of Cognitive Neuroscience, 22(7), 1614-1622.

Doughty, M. J., \& Naase, T. (2006). Further analysis of the human spontaneous eye blink rate by a cluster analysis-based approach to categorize individuals with 'normal'versus 'frequent'eye blink activity. Eye \& contact lens, 32(6), 294-299.

Durstewitz, D., \& Seamans, J. K. (2008). The Dual-State Theory of Prefrontal Cortex Dopamine Function with Relevance to Catechol-O-Methyltransferase Genotypes and Schizophrenia. Biological Psychiatry, 64(9), 739-749.

Elsworth, J. D., Lawrence, M. S., Roth, R. H., Taylor, J. R., Mailman, R. B., Nichols, D. E., et al. (1991). D1 and D2 dopamine receptors independently regulate spontaneous blink rate in the vervet monkey. J Pharmacol Exp Ther, 259(2), 595-600.

Frank, M. J., Loughry, B., \& O'Reilly, R. C. (2001). Interactions between frontal cortex and basal ganglia in working memory: A computational model. Cognitive Affective \& Behavioral Neuroscience, 1(2), 137-160.

Fukuda, K., Stern, J. A., Brown, T. B., \& Russo, M. B. (2005). Cognition, blinks, eyemovements, and pupillary movements during performance of a running memory task. Aviation Space and Environmental Medicine, 76(7), C75-C85.

Funahashi, S., Bruce, C. J., \& Goldman-Rakic, P. S. (1989). Mnemonic coding of visual space in the monkey's dorsolateral prefrontal cortex. J Neurophysiol, 61(2), 331-349.

Fuster, J. M., \& Alexander, G. E. (1971). Neuron Activity Related to Short-Term Memory. Science, 173(3997), 652-+.

Gramfort, A., Luessi, M., Larson, E., Engemann, D. A., Strohmeier, D., Brodbeck, C., et al. (2013). MEG and EEG data analysis with MNE-Python. Front Neurosci, 7, 267.

Groman, S. M., James, A. S., Seu, E., Tran, S., Clark, T. A., Harpster, S. N., et al. (2014). In the blink of an eye: relating positive-feedback sensitivity to striatal dopamine D2-like receptors through blink rate. Journal of Neuroscience, 34(43), 14443-14454.

Hall, A. (1945). The Origin and Purposes of Blinking. British Journal of Ophthalmology, 29(9), 445-467.

Hazy, T. E., Frank, M. J., \& O'Reilly, R. C. (2006). Banishing the homunculus: Making working memory work. Neuroscience, 139(1), 105-118.

Howes, O., McCutcheon, R., \& Stone, J. (2015). Glutamate and dopamine in schizophrenia: An update for the 21st century. Journal of Psychopharmacology, 29(2), 97-115.

Jongkees, B. J., \& Colzato, L. S. (2016). Spontaneous eye blink rate as predictor of dopaminerelated cognitive function-A review. Neuroscience and Biobehavioral Reviews, 71, 58-82. 
Jutkiewicz, E. M., \& Bergman, J. (2004). Effects of dopamine D1 ligands on eye blinking in monkeys: efficacy, antagonism, and D1/D2 interactions. J Pharmacol Exp Ther, 311(3), 10081015.

Kimberg, D. Y., D'Esposito, M., \& Farah, M. J. (1997). Effects of bromocriptine on human subjects depend on working memory capacity. Neuroreport, 8(16), 3581-3585.

Landau, S. M., Lal, R., O'Neil, J. P., Baker, S., \& Jagust, W. J. (2009). Striatal Dopamine and Working Memory. Cerebral Cortex, 19(2), 445-454.

Maffei, A., \& Angrilli, A. (2018). Spontaneous eye blink rate: An index of dopaminergic component of sustained attention and fatigue. International Journal of Psychophysiology, 123, 58-63.

Norn, M. S. (1969). Desiccation of the precorneal film. I. Corneal wetting-time. Acta Ophthalmol (Copenh), 47(4), 865-880.

Oh, J., Jeong, S. Y., \& Jeong, J. (2012). The timing and temporal patterns of eye blinking are dynamically modulated by attention. Hum Mov Sci, 31(6), 1353-1365.

Rac-Lubashevsky, R., Slagter, H. A., \& Kessler, Y. (2017). Tracking Real-Time Changes in Working Memory Updating and Gating with the Event-Based Eye-Blink Rate. Scientific Reports, 7.

Reddy, V. C., Patel, S. V., Hodge, D. O., \& Leavitt, J. A. (2013). Corneal Sensitivity, Blink Rate, and Corneal Nerve Density in Progressive Supranuclear Palsy and Parkinson Disease. Cornea, $32(5), 631-635$.

Roberts, A. C., Robbins, T. W., \& Weiskrantz, L. E. (1998). The prefrontal cortex: executive and cognitive functions: Oxford University Press.

Sawaguchi, T. (2001). The effects of dopamine and its antagonists on directional delay-period activity of prefrontal neurons in monkeys during an oculomotor delayed-response task. Neuroscience Research, 41(2), 115-128.

Seamans, J. K., \& Yang, C. R. (2004). The principal features and mechanisms of dopamine modulation in the prefrontal cortex. Prog Neurobiol, 74(1), 1-58.

Sescousse, G., Ligneul, R., van Holst, R. J., Janssen, L. K., de Boer, F., Janssen, M., et al. (2018). Spontaneous eye blink rate and dopamine synthesis capacity: preliminary evidence for an absence of positive correlation. European Journal of Neuroscience, 47(9), 1081-1086.

Siegle, G. J., Ichikawa, N., \& Steinhauer, S. (2008). Blink before and after you think: Blinks occur prior to and following cognitive load indexed by pupillary responses. Psychophysiology, 45(5), 679-687.

Sternberg, S. (1966). High-Speed Scanning in Human Memory. Science, 153(3736), 652-+.

Stewart, C. V., \& Plenz, D. (2006). Inverted-U profile of dopamine-NMDA-mediated spontaneous avalanche recurrence in superficial layers of rat prefrontal cortex. $J$ Neurosci, 26(31), 8148-8159. 
Swarztrauber, K., \& Fujikawa, D. G. (1998). An electroencephalographic study comparing maximum blink rates in schizophrenic and nonschizophrenic psychiatric patients and nonpsychiatric control subjects. Biological Psychiatry, 43(4), 282-287.

Taylor, J. R., Elsworth, J. D., Lawrence, M. S., Sladek, J. R., Roth, R. H., \& Redmond, D. E. (1999). Spontaneous blink rates correlate with dopamine levels in the caudate nucleus of MPTP-treated monkeys. Experimental Neurology, 158(1), 214-220.

Tharp, I. J., \& Pickering, A. D. (2011). Individual differences in cognitive-flexibility: the influence of spontaneous eyeblink rate, trait psychoticism and working memory on attentional set-shifting. Brain Cogn, 75(2), 119-125.

Tsubota, K., Hata, S., Okusawa, Y., Egami, F., Ohtsuki, T., \& Nakamori, K. (1996). Quantitative videographic analysis of blinking in normal subjects and patients with dry eye. Arch Ophthalmol, 114(6), 715-720.

Unsworth, N., Miller, A. L., \& Robison, M. K. (2019). Individual differences in encoding strategies and free recall dynamics. Quarterly Journal of Experimental Psychology, 72(10), 2495-2508.

Unsworth, N., Robison, M. K., \& Miller, A. L. (2019). Individual differences in baseline oculometrics: Examining variation in baseline pupil diameter, spontaneous eye blink rate, and fixation stability. Cogn Affect Behav Neurosci, 19(4), 1074-1093.

Van Slooten, J. C., Jahfari, S., \& Theeuwes, J. (2019). Spontaneous eye blink rate predicts individual differences in exploration and exploitation during reinforcement learning. Scientific Reports, 9.

Wager, T. D., \& Smith, E. E. (2003). Neuroimaging studies of working memory: A meta-analysis. Cognitive Affective \& Behavioral Neuroscience, 3(4), 255-274.

Westbrook, A., \& Braver, T. S. (2016). Dopamine does double duty in motivating cognitive effort. Neuron, 89(4), 695-710.

Xiao, J., Hays, J., Ehinger, K. A., Oliva, A., \& Torralba, A. (2010). Sun database: Large-scale scene recognition from abbey to zoo. Paper presented at the 2010 IEEE computer society conference on computer vision and pattern recognition.

Zhang, T., Mou, D., Wang, C., Tan, F., Jiang, Y., Lijun, Z., et al. (2015). Dopamine and executive function: Increased spontaneous eye blink rates correlate with better set-shifting and inhibition, but poorer updating. Int J Psychophysiol, 96(3), 155-161. 
a)

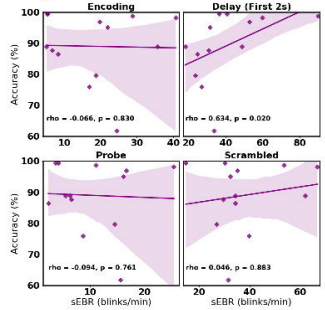

b)

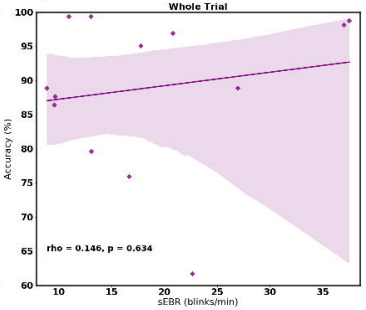




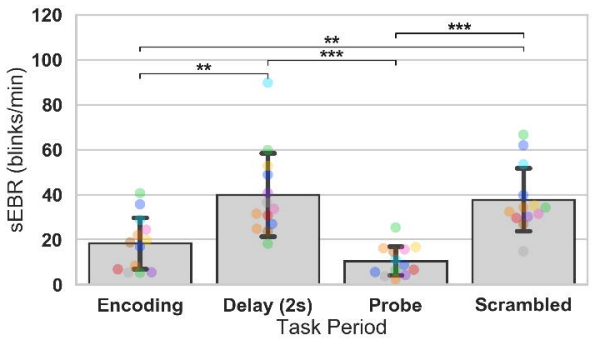


a)

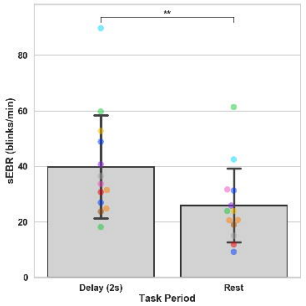

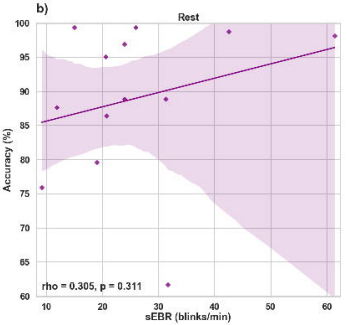




\section{a)}
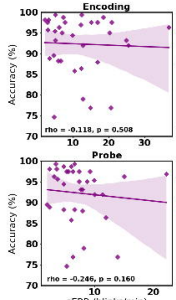

sEBR (blinksimin)
Delay (First 25)

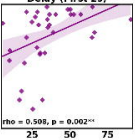

Scrambled

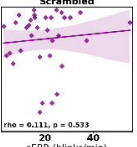

b)

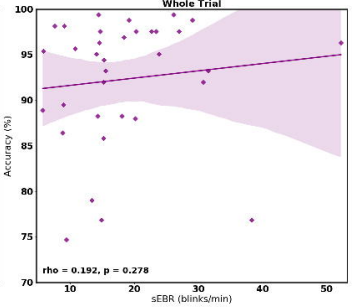




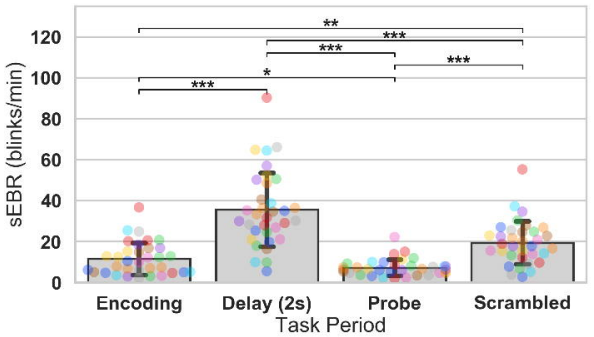


a)

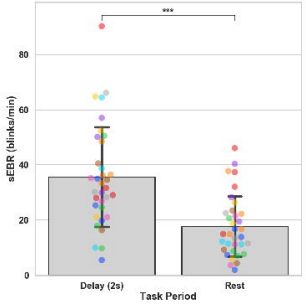

b)

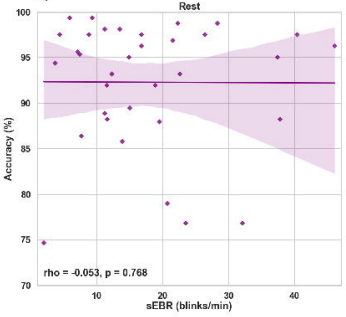


a)

Experiment 1 (Delay 2s)

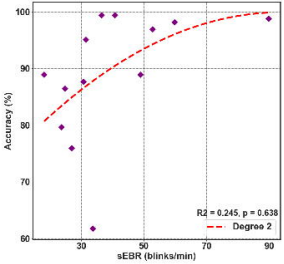

b)

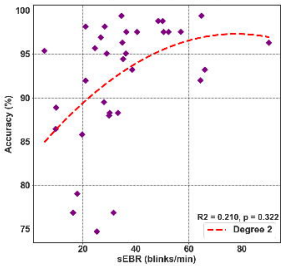

